

\title{
Capítulo 4 - Estado de la cuestió sobre estudios dialectales del quichua ecuatoriano
}

\author{
Luis Montaluisa Chasiquiza
}

MONTALUISA CHASIQUIZA, L. Estado de la cuestió sobre estudios dialectales del quichua ecuatoriano. In: La estandarización ortográfica del quichua ecuatoriano. Consideraciones históricas, dialectológicas y sociolingüísticas [online]. Quito: Editorial Abya-Yala, 2019, pp. 185-209. ISBN: 9789978-10-496-5. https://doi.org/10.7476/9789978104965.0005.

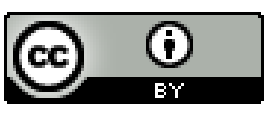

All the contents of this work, except where otherwise noted, is licensed under a Creative Commons Attribution 4.0 $\underline{\text { International license. }}$

Todo o conteúdo deste trabalho, exceto quando houver ressalva, é publicado sob a licença Creative Commons Atribição 4.0.

Todo el contenido de esta obra, excepto donde se indique lo contrario, está bajo licencia de la licencia Creative Commons Reconocimento 4.0 . 


\section{Capítulo 4 \\ Estado de la cuestión sobre estudios dialectales del quichua ecuatoriano}

En este capítulo hacemos un recorrido por los trabajos del quichua que de alguna manera aportan datos sobre la variación dialectal del quichua ecuatoriano. Esto permite visualizar el estado de las investigaciones dialectales de la lengua y la complejidad de un proceso de estandarización escrita.

La documentación del quichua ecuatoriano es bastante tardía. De esta variedad no ha llegado hasta nosotros documentos del siglo XVI ni XVII. Se conoce que en Quito y en otros lugares de la Presidencia de Quito, desde fines del siglo XVI, en los medios eclesiásticos se cultivaba el quichua para las campañas de evangelización. Pero los textos para el estudio, al parecer, eran traídos de Lima y seguramente eran los textos “estandarizados” por el Tercer Concilio Limense de 1583. De tal manera que existen vacíos históricos para conocer cómo eran las hablas del quichua del actual Ecuador en esos dos primeros siglos de la Colonia. Solamente unas pocas obras sobre el quichua ecuatoriano del siglo XVIII han llegado hasta nosotros.

Solo en el siglo XIX se imprimió un importante número de obras que contienen las hablas de este quichua. En ellas se visualizan ya las características del quichua ecuatoriano en cuanto a vocabulario y aspectos gramaticales; aunque a nivel de textos catequéticos y de la liturgia se mantuvieron los textos coloniales elaborados en el Perú. Tal es el caso del Padrenuestro, señalado por Ortiz (2001, p. 64). Este autor trae la ver- 
sión de esta oración recogida por el padre Gassó a fines del siglo XIX en la Amazonía ecuatoriana y publicada en 1898. El texto de esta oración es idéntico al del Segundo Concilio Limense de 1567, ratificado por el Tercer Concilio de 1583. En esa versión se mantiene el nosotros exclusivo cuando eso, al parecer, había ya desaparecido en las hablas ecuatorianas, pues en el Pater Noster redactado en Ecuador Runacunapac Rezana Libro del padre Julio Paris (1892/1913, p. 57), ya no aparece el exclusivo $<$ ñucaicu $>$, sino únicamente el inclusivo $<$ ñucanchic $>$, que es el único plural de primera persona que se usa en las hablas ecuatorianas.

Con estos antecedentes, a continuación, revisamos autores y obras que han contribuido al conocimiento de la realidad dialectal de las hablas quichuas del Ecuador.

\section{Época colonial}

Se ha mencionado que fray Jodoco Rique escribió la Doctrina cristiana y sermones en lengua peruana (Rivet y Créqui-Montfort, 1951, p. 25). El padre franciscano Agustín Moreno, historiador, al hablar sobre los documentos que fray Jodoco Rique habría llevado en su traslado de Quito a Pasto en 1570, a la edad de 72 años, después de haber vivido en Quito y haber dirigido el colegio de San Andrés para hijos de caciques indígenas, dice:

Otros manuscritos que seguramente llevó consigo deben ser los también perdidos "catecismo y Oraciones en Lengua Quichua", "De la consolación del varón religioso, que es enviado a predicar a los gentiles", "De las obras de Misericordia y de las prácticas del Evangelio", "Sermones varios en Lengua quichua y castellana", y aún otros manuscritos, de cuya existencia nos habla el sobrino del propio fray Jodoco, Adriano van Marselaer, en una síntesis biográfica de su tío, en la cual afirma que esas noticias las llegó a conocer a través de un franciscano que fue enviado preso al convento de Malina, después de haber vivido en el Perú y certificaba haberlo conocido (Moreno, 1998, p. 364). 
También se dice que el padre Martín de Vitoria escribió un Arte y vocabulario de la lengua del inca (Rivet y Créqui-Montfort, 1951, p. 26). Pero estas obras escritas en el siglo XVI no han llegado a ubicarse. Tampoco se sabe si existieron alguna vez. De encontrarse alguna de estas obras se podría observar la variante dialectal a la que representaban.

A nivel del Perú, hasta el presente, la primera gramática del quichua que se conoce es la del dominico fray Domingo de Santo Tomás. Este autor es consciente de la existencia de variaciones dialectales, pero no realizó ninguna propuesta de clasificación dialectal para las zonas del Perú que recorrió. En su texto se limita a señalar que el quichua era usado en un espacio muy extendido:

Principalmente, si añadiéramos a esto que es lengua que se communicava y de que se usava y se usa por todo el señorío de aquel gran señor llamado Guaynacapa, que se estiende por espacio de más de mil lenguas en largo y más de ciento en ancho. En toda la qual se usava generalmente della de todos los señores y principales de la tierra, y de muy gran parte de la gente común della (1560/1995, p. 9).

Un documento en donde se menciona al quichua relacionado con Quito es la gramática del doctor Alonso de Huerta. Esta obra cataloga al quichua ecuatoriano dentro del grupo Chinchaysuyo, en oposición al imperial cuzqueño, cuando señala:

Aunque la lengua Quichua y General del inga es una, se ha de advertir primero que está dividida en dos modos de usar en ella, que son, el uno muy pulido que se habla en el Cuzco, Charcas y demás partes de la provincia de arriba, que se dice Incasuyo. La otra lengua es corrupta, [que] la llaman Chinchaysuyo, [y] no se habla con la pulicía [el cuidado] y congruidad [congruencia] que los ingas la hablan. Y aunque hasta aquí no se ha enseñado documentos para hablar la lengua Chinchaysuyo, en este arte iré enseñando algunos para que se entienda la diferencia que hay de hablar entre las dos Provincias, que empiezan, la del inga desde Huamanga arriba, y la Chinchaysuyo desde allí abajo hasta Quito (1616/1993, p. 18). 
188

Según este dato, el QI estaría ubicado dentro de la variedad chinchaisuyo. Sin embargo, como se señaló en el capítulo anterior, esto no es exacto.

Juan de Figueredo (1619/1991), en la tercera edición del Arte de la lengua quichua del padre Diego Torres Rubio, trae un vocabulario chinchaisuyo de 135 palabras. De las cuales menos de la mitad son conocidas actualmente en el Ecuador.

Los documentos en donde se puede encontrar información sobre las hablas del quichua ecuatoriano corresponden recién al siglo XVIII. De este siglo se conoce la pastoral y el catecismo de Luis Francisco Romero, $14^{\circ}$ obispo de Quito, redactado en el dialecto del Ecuador, ${ }^{77}$ el 30 de agosto de 1725. Esta carta pastoral del obispo Romero contiene el Catechismo para instruir á los rudos en el misterio de la santissima eucharistia. El catecismo bilingüe quichua-castellano está conformado por 19 preguntas con sus respectivas respuestas.

Aparte de ese documento, del siglo XVIII se conocen otros tratados. Así, se tiene La breve instrucción o arte para entender la lengua común de los indios, según se habla en la provincia de Quito de 1753 atribuida a Tomás Nieto Polo del Águila, oriundo de Popayán quien fue provincial de los jesuitas de Quito al momento de la publicación de este documento.

El padre Grimm, en el "Prólogo" a su Vocabulario español-quichua en 1896, antes de transcribir dicho catecismo realiza un comentario útil para la dialectología del quichua ecuatoriano. Sobre la escritura de los fonemas /s/ y /š/, en la obra del obispo Romero dice:

77 El texto de la pastoral del obispo Romero de 1725 al que se ha tenido acceso es el que reprodujo Grimm en su obra de 1896. Se conoce que este catecismo también fue reproducido en la revista El Oriente Dominicano, publicada en Quito en la década de 1930, y en la Bibliographie des langues aymará et kičua (Rivert y CréquiMontfort, 1951, vol. 1, p. 141). 
189

Al mismo tiempo nos da este Catecismo una idea de la forma que el quichua tuvo en la primera mitad del siglo pasado. No hemos cambiado el modo de escribir las palabras quichuas, aunque muchas veces es diferente la escritura que hemos adoptado en la Gramática. Por lo que hace á la manera de escribir el quichua y á la sustitución de letras entre si, se ha dicho lo necesario en la Introducción á la Lengua Quichua. Tan solo observaremos para mejor inteligencia del Catecismo que los antiguos escribían los sonidos s y sh con el único signo s; así está escrito en nuestro Catecismo sina, etc., en lugar de shina, etc. (Grimm, 1896, pp. XXX-XXXI).

La observación de la escritura hecha por Grimm sobre la representación con la misma grafía $<\mathrm{s}>$ para /s/ y /š/, para el catecismo del obispo Romero, también es válida para el arte atribuido a Nieto Polo.

Otros textos del siglo XVIII son el Vocabulario de la lengua índica, de Juan de Velasco de cerca de 1787. El denominado "Anónimo de Praga", que lleva el título de Breve inst[r] uccion o arte para entender la lengua común de los indios. El Arte de la lengua jeneral de Cuzco llamada quichua que según Dedenbach-Salazar, reposa en el Archivo Nacional de Bogotá. ${ }^{78}$ Estos tres últimos sí emplean la grafía $<$ sh $>$ para representar a /š/.

\section{Época republicana}

A fines del siglo XIX y en las primeras décadas del siglo XX floreció un grupo de autores que escribieron sobre el quichua del Ecuador, los principales fueron: Lobato, Paris, Grimm, Cordero y Guzmán. De

78 El padre Gonzalo Ortiz Arellano, de la Orden de los Redentoristas, en su obra El quichua en el Ecuador: ensayo histórico-lingüistico pone en duda que el Arte de la lengua jeneral publicada por Dedenbach-Salazar sea del siglo XVII ni de ningún otro siglo del quichua ecuatoriano ( $c f$. Ortiz, 2001, pp. 41-43). Su argumentación es que la grafía $<$ sh $>$ solo aparecen en textos del quichua ecuatoriano de mitad del siglo XVIII y contiene un número importante de vocabularios que no son conocidos en los dialectos del Ecuador. 
190

ellos, para la cuestión dialectal, Cordero y Grimm son de mayor interés porque traen algunos datos al respecto. Los trabajos de Paris, Lobato y Guzmán no especificaron a qué dialecto representaban y por eso tienen menos utilidad para estudiar la dialectología. Parece que ellos trataron de hacer obras más generales que sirvan para la evangelización de los indígenas de toda la Sierra, en tanto que Cordero hizo su trabajo por un interés más cultural que religioso. Él era un civil ilustrado.

En la segunda mitad del siglo XX se incrementaron los estudios relacionados con el quichua ecuatoriano, por parte de ecuatorianos y extranjeros. A continuación presentamos los aportes que las diferentes obras han hecho para el estudio de las variaciones dialectales del quichua de Ecuador según el orden cronológico de su publicación.

\section{Luis Cordero}

Este autor elaboró un diccionario tomando como base el dialecto del Azuay (1892/1989). ${ }^{79}$ Aunque Cordero no establece comparaciones dialectales, su diccionario ha servido de referencia para autores como Parker al momento de realizar su clasificación dialectal del quichua ecuatoriano. El autor expresamente señala que su obra contiene el dialecto del Azuay.

En las breves nociones gramaticales concernientes al idioma quichua que pone al inicio de su obra, señala que es necesario emplear las letras $<\mathrm{zh}, \mathrm{z}>$ para los sonidos del quichua que se habla en esa región. En su léxico incluye 26 palabras que comienzan con $<\mathrm{zh}>$ y 14 con $<\mathrm{z}>$. Estas, al parecer, son sustratos del cañari, excepto $<$ zarca $>$ que proviene del hispano-árabe <zarco, zarca > 'persona de ojos azules'.

79 Luis Cordero nació en Déleg (prov. de Cañar) y posteriormente fue a Cuenca (prov. de Azuay), por lo cual su diccionario es representativo de las hablas de la Sierra sur del Ecuador. 


\section{1}

\section{Juan M. Grimm}

El sacerdote alemán Grimm escribió un par de obras sobre el quichua ecuatoriano a fines del siglo XIX (1896) y comienzos del XX (1903). Escribió una gramática que incluye un vocabulario quichua-español y un vocabulario español-quichua. Además, publicó un vademécum para párrocos de indios quichuas. La primera obra es la que nos interesa para la dialectología. Él dice que cuando ya estaba impresa la primera sección —esto es el vocabulario quichua-español— encontró nuevos términos quichuas, particularmente del Oriente, que los incluyó como apéndice antes de la sección español-quichua (La lengua quichua: dialecto de la república del Ecuador, 1896). Mientras los otros autores presentan sus gramáticas y diccionarios sin preocuparse por las variaciones dialectales, Grimm hace mención a algunas diferencias entre las hablas de la Sierra y la Amazonía. Así, en la introducción a su gramática dice:

El dialecto de Quito hablan todavía casi medio millón de indios entre las dos cordilleras de los Andes desde el $4^{\circ}$ grado de latitud meridional hasta $1 / 2$ grado de latitud septentrional y en las llanuras superiores del río Napo; pero con la diferencia de que los indios del Napo hablan el dialecto de Quito en su forma antigua, aunque ya empiezan también a sustituir la $\mathbf{p}$ con la $\mathbf{b}$ y la $\mathbf{t}$ con la $\mathbf{d}$; mientras los de entre las cordilleras lo hablan adulterado. Esta decadencia consiste primero en que el idioma degenera de suyo mismo: si comparamos, por ejemplo, el idioma como lo enseña la "Breve instrucción para entender la lengua común de los indios, según se habla en la provincia de Quito", con el actual, observamos ya perdido en siglo y medio el uso del plural inclusivo y exclusivo y disminuido mucho el uso de la conjugación de complemento personal y de las formas verbales que sirven para expresar los pronombres posesivos. Además los indios han perdido el conocimiento de muchas palabras del dialecto antiguo de Quito. Fuera de esto emplean apócopes, principalmente en el gerundio (Grimm, 1986, p. VI).

En esta obra de Grimm también se encuentra una compilación de algunas piezas literarias en quichua, principalmente las escritas por Luis Cordero, con notas de pie de página, en las cuales señala algunas 
192

especificidades dialectales del habla quichua de la provincia de Azuay, que está en la Sierra sur del Ecuador. Además, incluyó un apéndice con léxico propio de la Amazonía ${ }^{80}$ y alguna información de variación dialectal entre el habla de la Sierra y el dialecto de Tena, en la Amazonía. Así, por ejemplo, nos da a conocer que en el habla de Tena, en el siglo XIX, ya se empleaba la variante [ra] para el morfema del acusativo $\{$-ta $\}$.

\section{Agustín María León}

En la década de 1930, el padre León publicó por entregas sucesivas en la revista El Oriente Dominicano el Compendio de gramática quichua, basado en el quichua de Canelos (río Bobonaza, prov. de Pastaza, Amazonía ecuatoriana). Sobre la dialectología, el autor dice: "Es el mismo dialecto de los pueblos indígenas de la sierra, con algunas excepciones que van anotadas en su lugar" (León, 1937, p. 202). Entre algunas de las notas sobre la variación dialectal señala la fusión del morfema independiente de afirmación $\{-\mathrm{mi}\}$ con el verbo 'ser' $\{\mathrm{kay}\}$, produciéndose un apócope del morfema independiente y una aféresis del verbo, con lo cual, por ejemplo, la expresión <allimi kan> 'está bien' se transforma en [aliman]. También hace notar la síncopa del morfema de pasado $\{-$ rka- $\}$ para transformarse en [ra]. Al respecto, el padre León señala: "En Canelos y pueblos del Bobonaza dicen: mani; mangui; man y usan el carani en lugar de la formula general carcani, y eluden siempre la c después de r" (León, 1937, p. 241).

\section{Ellen Ross}

Esta misionera evangelista había aprendido el quechua en Bolivia, luego vino al Ecuador, donde aprendió el quichua de la provincia de Chimborazo y recorrió algunos lugares de la Sierra ecuatoriana.

80 El léxico de la Amazonía registrado por Grimm dice haberlo tomado de un Vocabulario inga-español, cedido por los padres de la Compañía de Jesús de Quito. Era de esperarse que este texto estuviera en la biblioteca de los jesuitas de Cotocollao, pero hasta el presente no se ha podido localizar. 


\section{3}

Ella escribió dos obras relacionadas con el quichua. Según Richard A. Aschmann, ${ }^{81}$ quien reeditó en versión electrónica la obra de Ross en 2009, vivió solo un año en el Ecuador, en 1958 o 1959.

Ross elaboró un método bilingüe quichua-inglés y también un diccionario quichua-inglés, basándose en el dialecto de la provincia de Chimborazo, para que los religiosos evangélicos pudiesen aprender la lengua indígena.

En su método bilingüe, la autora enumera siete dialectos quichuas de la Sierra ecuatoriana: Colta, Pulucati y Caliata (prov. de Chimborazo), Calderón (prov. de Pichincha), Agato (prov. de Imbabura y asociado con el de Cayambe que está en la prov. de Pichincha), Cuenca (prov. de Azuay) y Saraguro (prov. de Loja). En la presentación de su método señala algunos ejemplos de variaciones dialectales al decir:

The dialect variation which your language helper points out will seem much less confusing if you learn to sort them out into several different categories. Some will be merely a sound level (for example, a certain sound used in CHQ may always be changed into another sound in Imbabura), Others will be on the suffix level, as when a certain suffix is pronounced one way in Colta but another way in Pulucate. Still other changes will be on the level of vocabulary, as when a word that is often used in Colta is substituted by another in Pulucate. Still others will be on the level of grammar, and others in the matter of style. In this lesson only the first two kinds of dialect variations appear.

$\mathbf{L l}$ is in CHQ, Imbabura, Salasaca and Pichincha always pronounced like the $z h$ in "azure".

81 Aschmann realizó la traducción al castellano de la gramática quichua-inglés de Ross en 2009. Esta obra, antes que una gramática, es un método para aprender la lengua por parte de personas de habla inglesa. Sobre la obra dice la autora en su prólogo: "This course has been prepared with a view to providing a concise but comprehensive teaching guide for missionaries wishing to learn Ecuadorian Highland Quichua” (Ross, 1958-1959/2009, p. v). 
194

Imbabura also pronounces it this way. However, in many words that have $\mathbf{l l}$ in $\mathrm{CHQ}$, Imbabura has a simple $\mathbf{l}$. This occurs when the CHQ 11 is followed immediately by the vowel $\mathbf{I}$, or by a consonant. Thus, CHQ alli "good" is ali in Imbabura, and CHQ allcu "dog" is alcu in Imbabura. The $\mathbf{c}$ in cangui and Cana becomes $\mathbf{g}$ in Pichincha and Salasaca.

The suffix -lla occurs in allilla, "Well", (although for the present it is not being studied in detail). In Pulucate, Caliata and Flores (in Chimborazo), and in Pichincha it is pronounced (and spelled) -la (Ross, 1958-1959/2009, pp. 1-2, 1-3).

En su obra, la autora estableció en primer lugar el quichua de la Sierra centro (CHQ) integrado por Colta, Pulucate y Caliata. En segundo lugar señaló el dialecto Calderón y el dialecto Imbabura/Cayambe. ${ }^{82}$ En tercer lugar, el quichua de la Sierra sur (SHQ) integrado por Azuay y Saraguro. Ella no hace referencia a otras hablas de la provincia de Chimborazo ni tampoco a las de las provincias de Cotopaxi, Bolívar y Cañar. De Tungurahua solo menciona a Salasaca, que apenas es una parte de esta provincia. Sin embargo, la clasificación de los dialectos quichuas señalada por Ross fue un antecedente de la clasificación del quichua ecuatoriano en dialectos centrales y dialectos no centrales que posteriormente hicieron algunos lingüistas en la siguiente década.

\section{Gary Parker}

En 1969, Parker presentó un estudio de la evolución de lo que él denomina QA en el cual muestra una clasificación dialectal tentativa del quichua ecuatoriano. Esta clasificación se basó en la información de trabajos anteriores relacionados con el quichua de Ecuador (Cordero, 1892/1955; Orr, 1965; Paris, 1892/1924; Ross, 1958-1959/2009). Sobre los dialectos de Ecuador, Parker dice:

82 La autora no denomina - como podría haberse esperado- NHQ a los dialectos de Calderón, que están en el centro de la provincia de Pichincha, y al de Agato/ Cayambe (Agato está en la provincia de Imbabura y Cayambe al norte de la provincia de Pichincha). 
195

Chart III shows a tentative identification and subgruping of Ecuadorian dialects based mainly on data from Cordero 1955 for southern Highland, Ross 19?? and 196? For Central and Northern Highland, and Orr and Wrisley 1965 for Jungle. So great are both the amount of dialect diversification in Ecuador and the size of the geographical area involved that much systematic dialectological field work must be carried out before a comprehensive statement of the Ecuadorian Quechua situation can emerge (Parker, 1969, p. 154).

A partir de lo que denomina un "proto-ecuatoriano", Parker separa dos ramas de dialectos: Sierra y Amazonía. Luego a los dialectos de la Sierra los clasifica en dos subramas: central-norte y sur. Asimismo, al central-norteño lo divide en norteño y central. Luego, al norteño lo clasifica en Agato y Calderón, y al central en Colta y Pulucate-Caliata. Finalmente, estos últimos son subdivididos en Pulucate y Caliata. Además, el sur es clasificado en Saraguro y Azuay. Por otra parte la rama de la Amazonía se subdivide en Bobonaza-Tena y Limoncocha. Finalmente, Bobonaza-Tena se subdivide en dos.

Figura 8

Clasificación dialectal del quichua ecuatoriano



Fuente: Parker, 1969, p. 157; 2013, p. 151 
196

Como se puede apreciar en la figura, en el caso de la Amazonía, Parker se apoyó en la información de Carolyn Orr (1965), en tanto que para la Sierra tomó en consideración los trabajos de Ellen Ross. Lo interesante de la propuesta de Parker es que intenta hacer una clasificación integral, tanto de los dialectos de la Sierra cómo los de la Amazonía, que hasta ese entonces se habían tratado por separado. Sin embargo, por obvias razones de información, en esta propuesta - principalmente en el caso de la Sierra- solo tomó en consideración tres hablas de la provincia de Chimborazo (Colta, Pulucate y Caliata), una de la provincia de Imbabura (Agato), una de la provincia de Pichincha (Calderón), una de Azuay y una de Loja (Saraguro), quedando por ubicar las hablas de las provincias de Cotopaxi, Tungurahua y Bolívar, el sur de la provincia de Chimborazo y la provincia de Cañar.

A pesar de las limitaciones de la época, un aspecto importante a tomar en cuenta es que poco a poco se fue consolidando la idea de clasificar los dialectos de la Sierra en: norteño, central y sureño. Esto será retomado por Peter Cole (1985) y reformulado después por otros autores como Carpenter (1982).

\section{Alfredo Torero}

En su trabajo "Los dialectos quechuas" (1964/2003), con la información bibliográfica de Paris (1892/1924), Grimm (1896), Cordero (1892/1955) y Guzmán (1920), ubicó al quichua (quechua) ecuatoriano, junto con el Ingano de Colombia, en el grupo IIB. Señaló que este dialecto en lo fonológico y lo gramatical estaba a un paso intermedio entre el IIA y IIC. ${ }^{83}$ Observó que una de las características de la variedad ecuatoriana es la sonorización de las oclusivas y africadas orales tras /n/ en el interior de palabra, y señaló otras características como: 
Se habla en los valles interandinos y en el Oriente ecuatorianos. Tiene numerosas variedades cuyas características separadoras no conocemos suficientemente. Ha puesto en desuso los morfemas de persona poseedora que expusimos páginas antes, maneja solo un morfema de plural personal, sin distinción de "inclusivo" y "exclusivo" [...]. Para el "durativo" emplea el morfema /-ku-/. Parecen estar cercanamente emparentadas con los dialectos ecuatorianos las hablas de Colombia (donde el "Ingano" no distingue $* / \mathrm{s} / \mathrm{y} / \mathrm{sh} /, \mathrm{y}$, tal vez, las de la provincia peruana de Maynas (Loreto) utilizadas a lo largo del río Napo (Torero, 1964/2003, p. 55).

De las características del quichua ecuatoriano señaladas por Torero, la sonorización de las oclusivas y africadas después de nasal es parcialmente exacta, pues no se da en todo el léxico de Cañar, Azuay y Loja; pues se mantiene la sordez en términos como [inti] inti 'sol', [yanta] yanta 'leña'. El fonema / $\mathrm{k}$ / es el que más se sonoriza después de $/ \mathrm{n} /$, pero $/ \mathrm{p} / \mathrm{y} / \mathrm{t} / \mathrm{se}$ mantienen como sordas, principalmente en los morfemas. La sonorización de las africadas /č/, y / $\$$ / después de nasal/n/, solo se da en algunos dialectos de las provincias de Imbabura, Cotopaxi, Tungurahua y Chimborazo. En los demás dialectos no se sonorizan las africadas.

En cuanto a la no distinción entre plural inclusivo y exclusivo, la afirmación de Torero es correcta, según ya había sido anotado por autores como Grimm (1896). El uso del morfema \{-ku\} como durativo es igualmente correcta. Por lo demás, el autor menciona que hay varios dialectos, pero no señala cuales son. Textualmente dice:

Ecuador-Colombia. Se presenta con numerosas variedades que no se han deslindado aun suficientemente [...]. Las variedades del oriente ecuatoriano han avanzado al parecer hasta el curso alto y medio del río Napo y quizá hasta otros afluentes septentrionales del río Amazonas (Torero, 2002, pp. 83-84).

En la bibliografía de su obra de 1964 no están las obras de Ellen Ross y en la bibliografía de sus trabajos posteriores, incluida su última obra de 2002, tampoco se menciona las obras de Ross o Carolyn Orr. 
198

Posiblemente debido a que no pudo revisar los trabajos de esas autoras, Torero no propuso una clasificación dialectal interna para el quichua ecuatoriano. A diferencia de Torero, Parker sí conoció los textos de Ross y Orr, y los tomó en cuenta en su propuesta de clasificación dialectal.

Torero ubicó a todas las hablas del quichua ecuatoriano junto con el ingano de Colombia en el grupo IIB. Esto se puede apreciar en la Figura 3 (cf. cap. 3).

\section{Carolyn Orr}

Esta autora publicó en 1965 el Vocabulario quichua del Oriente, en cuya presentación ya mencionaba que existían tres dialectos en la Amazonía ecuatoriana que son: Bobonaza, Tena y Limoncocha. En 1978 publicó un pequeño folleto con los resultados de su investigación sobre dialectos quichuas, elaborado a partir de un borrador de 1973. En este trabajo clasifica al quichua del Ecuador en dialectos de la Amazonía y dialectos de la Sierra. Su estudio dialectal pone énfasis en los tres de la Amazonía ya mencionados (ver cita más abajo). De la Sierra no hace una clasificación dialectal, pero en un glosario de 284 palabras que incluye en su folleto, trae variaciones fonéticas de ese corpus léxico en las provincias de Imbabura, Pichincha, Chimborazo, Cañar y Azuay que están en la Sierra y de las provincias de Pastaza y Napo en la Amazonía. ${ }^{84}$ Sobre los dialectos de la Amazonía dice:

En el Oriente ecuatoriano, hay tres principales dialectos quichuas: uno en la provincia de Pastaza, y los otros dos en la provincia del Napo. Al primero lo llamaremos Pastaza; se localiza alrededor del Puyo, y a lo largo de los ríos Bobonaza, Conambo y Curaray. El segundo lo llamaremos Tena; se ubica alrededor de Tena, Archidona y Arajuno, y por el bajo Napo hasta Yurallpa. Al tercer dialecto lo llamaremos Napo; se extiende

84 Con posterioridad a 1978, fecha de publicación de la obra de Orr, la provincia de Napo fue dividida en dos nuevas provincias: Sucumbíos y años después Orellana. 
desde Loreto y Aquila por los ríos Payamino y Coca, y desde la desembocadura de dichos ríos en el Napo, y hasta Rocafuerte [se refiere a Nuevo Rocafuerte, pues hay un Rocafuerte que está más abajo, en el mismo río Napo, pero en territorio peruano]. Este tercer dialecto se habla también a lo largo de los ríos Putumayo y bajo Aguarico (Orr, 1978, p. 6).

En su trabajo de 1978, Orr sustituyó la nomenclatura de los dialectos de la Amazonía que había propuesto en 1965. En aquella época los había denominado dialectos Bobonaza, Tena y Limoncocha. En este nuevo trabajo ella habla de tres dialectos que son: Pastaza, Tena y Napo. Reemplazó Bobonaza por Pastaza, Limoncocha por Napo y mantuvo el nombre del dialecto de Tena. Estos cambios de denominación de los dialectos muestran una mejora en la precisión de su ubicación. Efectivamente, el nombre de Bobonaza no representaba a las hablas de los ríos Conambo y Curaray que son afines y están también en la provincia de Pastaza. Sin embargo, el nombre de Pastaza tampoco es exacto, pues Arajuno está en la provincia del río Pastaza, pero es un habla más afín con Tena, que está en la provincia de Napo. Igualmente, el cambio de nombre del dialecto de Limoncocha por Napo era necesario, pues sus habitantes tienen el mismo dialecto que los de Tena, por lo tanto, había que buscar un nuevo nombre para la variante que estaba en el río Napo medio. Con todo, el término Napo tampoco representa a las hablas quichuas del río Putumayo. En consecuencia, está pendiente buscar nombres más apropiados para denominar a los dialectos de la Amazonía. En los estudios lingüísticos, con frecuencia, existen problemas entre la jurisdicción política y la realidad dialectal.

Las variaciones morfofonémicas del quichua de la Amazonía ecuatoriana estudiadas por Orr tienen que ver con la conducta de los siguientes sufijos: $\{$-pi\} 'locativo', $\{-\mathrm{ku}$ - $\}$ 'progresivo', $\{$-shka\} 'indicador de acción completa', $\{$-shpa $\}$ 'mismo sujeto', $\{-\mathrm{kppi}\}$ 'cambio de sujeto', $\{$-yuk $\}$ 'posesor', \{-pa\} 'posesivo', \{-kuna\} 'plural', $\{-y\}$ 'imperativo', $\{$-naya- $\}$ 'desiderativo', \{-ta\} 'complemento directo', $\{$-rka- $\}$ 'pretérito', $\{$-nkuna $\}$ 'tercera persona plural', $\{$-naku-\} 'recíproco', $\{$-nkapak\} 'propositivo'. No obstante de dicho avance, quedaba por investigarse las variaciones de la 


\section{0}

mayor parte de los morfemas del quichua amazónico ( $c f$. cap. 5 para las variaciones amazónicas de los morfemas).

\section{Pieter Muysken}

Este autor viene estudiando el quichua de Ecuador desde mediados de la década de 1970. Muysken sugiere que su trabajo es el comienzo de una línea de investigaciones que se podría realizar en el futuro, sobre la morfofonémica; cuando dice:

The following section presents an informal account of the different phonological changes which the suffixes of EcQ have undergone. In most cases, such as voicing and vowel changes, similar but less regular changes also affect the EcQ lexicon. These will not enter into consideration here (Muysken, 1977, p. 10).

Hizo algunas aportaciones al estudio dialectal del quichua de la Sierra ecuatoriana. En su obra presenta las variantes morfofonémicas en el dialecto de la Sierra centro de los morfemas: $\{$-pi $\},\{$-kama $\},\{-k u n a\},\{-$ ta $\}$, $\{$-pak $\},\{$-man $\},\{$-rka $\},\{$-gri $\},{ }^{85}\{$-shpa $\},\{\mathrm{kpi}\}$ y $\{$-pish $\}$. Aparte de ello, señala la variación de 11 vocablos quichuas: "sumak, kunan, yawar, hayak, illakta, yachak, ñan, chay, shamuy, tukuy, ñaubay” (cf. Muysken, 1977, pp. 10-14).

\section{Laurence K. Carpenter}

A partir de la década de 1970 se realizan algunas tesis de lingüística en universidades extranjeras, sobre algunas hablas locales del quichua ecuatoriano. Así, Beukema (1975) realiza una descripción gramatical del quichua de Chimborazo como tesis para la Universidad de Yale. Lombeida-Naranjo (1976) realiza un estudio fonológico de la Sierra como tesis doctoral para la Universidad de Texas. Igualmente, Consuelo Yánez, de la PUCE, realiza en esta década estudios sobre la fonología del quichua en los dialectos de Chimborazo, Imbabura y Loja.

85 Muysken presenta como /-gri/ al morfema $\{-k r i\}$ (1977, p. 14). 
En este contexto, Carpenter llega al Ecuador procedente de Estados Unidos, como integrante del Cuerpo de Paz. Inicia su aproximación al quichua con Louisa Stark a inicios de la década de 1970. Aparece como coautor de El quichua de Imbabura: una gramática pedagógica. Este texto estuvo destinado a la enseñanza del quichua y fue elaborado con Stark (1973).

En el prefacio de dicho texto, los autores señalan que existen cinco subdialectos en la provincia de Imbabura:

Para el desarrollo de este libro nos hemos basado en el dialecto Quichua de la Provincia de Imbabura, ubicada en la Sierra norte del Ecuador. Este dialecto se divide en cinco subdialectos los mismos que se hablan en las siguientes zonas: 1. Desde Cayambe por San Pablo y desde el este de Imbabura a Angla, Zuleta, Angochahua y Rinconada, y desde estas comunidades hasta Mariano Acosta y Pimanpiro; 2. La comunidad de San Rafael; 3. La zona de San Rafael al norte hasta San Roque en el lado oriental del Río Ambi; 4. Al norte de San Roque hasta San Antonio de Ibarra en el lado oriental del Río Ambi; 5 . Al norte de San Rafael y al oeste del Río Ambi por las faldas de Cotacachi. Cada zona se distingue de las otras por diferencias menores en cuanto al habla (pronunciación, gramática y vocabulario) y en la manera de vestir (Stark y Carpenter, 1973, p. I).

Aquí los autores señalan los lugares que integran los subdialectos de Imbabura, pero no especifican en qué consisten las diferencias dialectales, se limitan a decir que su gramática pedagógica se basa en el subdialecto 3, particularmente en el habla de Miguel Andrango Concha y Carlos Conterón Córdova, de Agato e Ilumán, respectivamente, y que hay diferencias de pronunciación, gramática y vocabularios, pero no señalan ni un solo ejemplo concreto de tales variaciones. A pesar de ello, el dato de que al interior de una misma provincia existen varios dialectos plantea la inquietud de investigar el mayor número de ellos para tener un mapa dialectal más completo de la región. 


\section{2}

Figura 9

Los cinco subdialectos del quichua de Imbabura

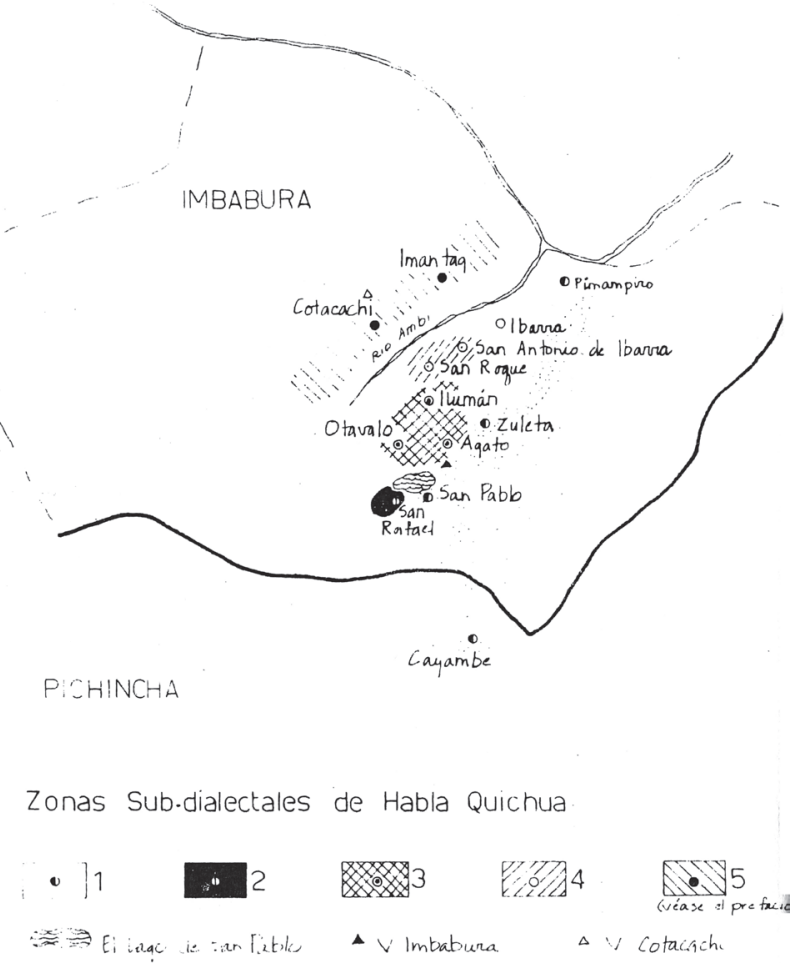

Fuente: Stark y Carpenter, 1973, p. VI

Después de haber trabajado con Louisa Stark, Carpenter visitó otros lugares de habla quichua, principalmente de la Sierra y la Amazonía, para realizar su tesis doctoral. Los lugares recorridos son las provincias de Imbabura, Pichincha, Cotopaxi, Tungurahua, Chimborazo, Bolívar, Cañar, Azuay, Loja, Napo y Pastaza. En su tesis ofrece una síntesis de sus investigaciones bibliográficas, principalmente del período 19501980, luego se centra fundamentalmente en dos aspectos: la descripción de tres niveles de la lengua (fonología, morfología y sintaxis) basándose 


\section{3}

fundamentalmente en el habla de la provincia de Imbabura — que fue su centro de operaciones- y su estudio de las variaciones dialectales.

Sobre los niveles de la lengua, Carpenter repite el trabajo que ya otros autores revisados por él habían hecho. Su cuadro fonológico ( $p h o-$ nemic chart) presenta 33 unidades, pero sin incluir pares mínimos para justificarlos, de las cuales cuatro aparecen entre paréntesis porque considera que provienen del español. Estos son: /b, d, g, ř/. Incluye también un fono fricativo bilabial sordo y una unidad oclusiva palatal sorda distinta de /č/ y de / $\$$ /, sin justificar con pares mínimos. El número elevado de fonemas señalados por él dista del número que realmente tiene el quichua ecuatoriano, pues incluyendo los fonemas aspirados y los de sustrato /z, ž/, apenas llegarían a 24 . De otro lado, en lo relacionado con la morfología, hace una descripción de los morfemas de la lengua. De cada morfema señala dos o tres ejemplos para mostrar su uso, pero sin preocuparse por las variaciones morfofonémicas. Por último, en lo que corresponde a la sintaxis, presenta los constituyentes de la oración, la expansión del nombre y la expansión del verbo.

En cuanto a la variación dialectal, ${ }^{86}$ afirma que los 14 dialectos del quichua ecuatoriano se puede agrupar en dos: el central y el no central. De estos dos grupos, el dialecto denominado central es el más innovador.

Within Ecuador, Quichua exhibits variation at the phonological, morphological, syntactic, and lexemic levels. There are at least 14 dialects of Ecuadorian QIIB subdivided into two major groups, central and non-central. The central varieties exhibit a substantial amount of innovations and variation and most of these dialects probably have their origin in the varieties of Quechua spread by de Chinchay (Carpenter, 1982, p. 41).

86 En el texto de Carpenter (1982), los capítulos que se refieren a los aspectos dialectales y a la sintaxis son los más cortos de todos. Y a pesar de que en el capítulo sobre la dialectología hay mapas de algunos alófonos de los fonemas, incluye muy pocos ejemplos ilustrativos de tales variaciones dialectales. 


\section{4}

Figura 10

Dialectos ecuatorianos según Carpenter

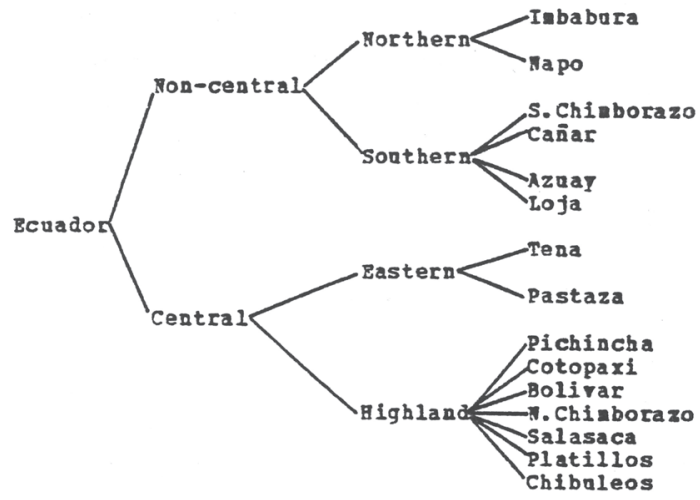

Fuente: Carpenter, 1982, p. 42

Figura 11

Dialectos del quichua ecuatoriano según Carpenter



Fuente: Carpenter, 1982, p. 21 


\section{5}

Sobre los "aspectos de dialectología", sostiene que los quichuahablantes de los dos dialectos mayores (central y no central) prefieren comunicarse no en quichua, sino en español.

When Quichua speakers from these two major dialect areas attempt to communicate across the major division boundaries, it is usually carried out in Spanish (or another indigenous language) rather than Quichua. In some cases, the central and the non-central dialects are mutually unintelligible across the major boundary divisions. For example, consider the following utterances, both glossed as 'it is well.'

ali.mi (Imb.)

[alími]

Alli.lladi mi.shka (Tsl.)

[ažižadi míška]

With such variation, bilingual Quichua speakers from these areas consider the other dialects of Quichua to be 'scrambled,' 'mixed.' Or 'not for understanding.' Such bilingual speakers will communicate in Spanish when possible (Carpenter, 1982, pp. 355-356).

La afirmación de Carpenter de que los dos dialectos el central, y el no central son mutuamente ininteligibles no es muy exacta, principalmente a nivel de la Sierra, pues en los cursos de capacitación pedagógica del sistema intercultural bilingüe se ha podido constatar que es posible una intercomprensión, aunque asimétrica. Los hablantes de los dialectos denominados centrales comprenden más a los hablantes de los dialectos no centrales que viceversa. De todas maneras, se ha podido apreciar que luego de unos días de contacto entre los hablantes de estos dos dialectos sí pueden llegar a comprenderse, aunque les resulte bastante extraño el habla del otro interlocutor.

Carpenter presenta 13 mapas en los cuales muestra los lugares de algunas variaciones dialectales a nivel fonético. El autor se limita a presentar los fenómenos de la sonorización de las oclusivas sordas, las reducciones fonéticas, la aspiración y fricativización, la realización de las líquidas, la haplología y la metátesis. A cerca de la variación fonológica dice: 


\section{6}

Phonological variation in the non-central dialects are, for the most part, contemporary reflexes of earlier forms of the language. Thus sound changes are merely allophonic pronunciation differences, and the basic underlying phonemic structure has not been altered. For example, the voicing of occlusives after stops, the realization for the word-final velar $/ \mathrm{k} /$, and the consonantal weakening are manifested in various stages in Ecuador. Regarding voicing of stop consonants after nasals, consider the following examples.

[kamba] [kampa] /kanpa/ 'yours'

[kanda] [kanta] /kanta/ 'to you'

[kanga] [kanka] /kanka/ 'and you' (Carpenter, 1982, p. 357).

Como una ilustración de los mapas de este autor, a continuación presentamos el mapa de variación de lo que él denomina "Realization of liquids" (Carpenter, 1982, p. 368). En ellas están comprendidos los fonemas $/ \lambda / \mathrm{y} / \mathrm{r} / .^{87}$

Figura 12

Realización de los sonidos /I/ y /r/ según Carpenter

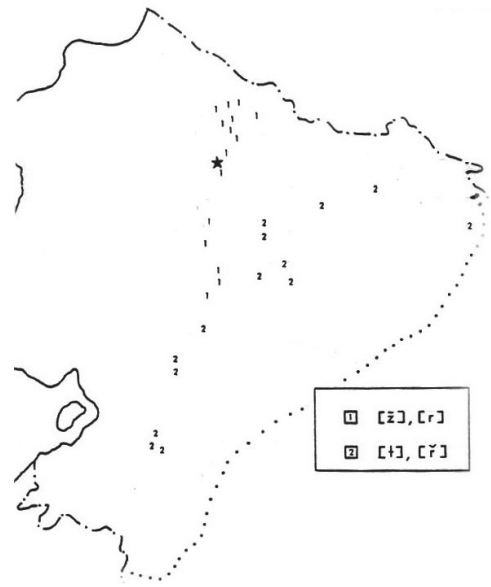

Fuente: Carpenter, 1982

87 Carpenter representa al fonema $/ \lambda /$ con el símbolo $ł$. 


\section{7}

Tales mapas, sin embargo, no resultan muy ilustrativos porque no señalan las provincias en las cuales se manifiestan los fenómenos estudiados. Esto dificulta la ubicación exacta de las variantes en el mapa. Como se verá más adelante, la variación del fonema $/ \lambda /$ se debe estudiar no solamente a nivel del léxico, sino también del morfema $\{-\lambda \mathrm{a}\}$. Esto permitirá una mayor aproximación en la representación objetiva de la variación dialectal.

Sobre los fenómenos fonéticos como reducción, metátesis, etc., presenta los mapas con los lugares donde ocurren, pero sin señalar ejemplos concretos. En cuanto a la morfología, solo estudia las variaciones de los morfemas $\{-$ naku $\},\{$ pak $\},\{$ rka\}. En lo relacionado con las variaciones sintácticas solo menciona el uso de $<$ kikin $>$ y $<$ kan $>$ para la segunda persona. Dice que en la provincia de Imbabura se hace una distinción para la segunda persona, entre $<$ kikin $>$ 'usted' $\mathrm{y}<\mathrm{kan}>$ 'tú', mientras que en los demás dialectos no se conoce dicha diferencia. La afirmación de Carpenter sobre esto es parcial, pues < kikin $>$ también se usa en las provincias de Pichincha, Cotopaxi, Tungurahua, Bolívar y Chimborazo. El uso de $<$ kikin $>$ con el significado de 'usted' se encuentra ya en Yánez y Jara (1975). Sobre las variaciones lexicales, el autor presenta que hay nueve formas distintas de pronunciar la palabra $<$ atallpa $>$ 'gallina', sin embargo, hay que mencionar que en verdad existen más de veinte formas diferentes de pronunciación de esta palabra, por lo que lo manifestado por Carpenter es solo parcial. Finalmente, la limitación más importante de Carpenter es que no hizo diferencia entre las variaciones fonéticas del léxico y las variaciones fonéticas de los morfemas, el estudio diferenciado de estos dos componentes resulta muy importante para el estudio dialectal del quichua. Por lo demás, el autor no presenta criterios explícitos en los que se basó para su clasificación dialectal.

\section{Rodolfo Cerrón-Palomino}

Cerrón-Palomino (1987, cap. VIII) propone la clasificación de los dialectos de la Sierra ecuatoriana en norte, centro y sur. Al referirse al quichua de la Sierra dice: 


\section{8}

En este mismo subgrupo se pueden distinguir, a su vez, tres subramas, correspondientes a las regiones Norte, Centro y Sur. Los dialectos del norte, formados básicamente por los de Imbabura y Pichincha, aunque mantienen la $/ \mathrm{t}^{\mathrm{h}} /$ (de muy escasa ocurrencia), han cambiado la / $\mathrm{p}^{\mathrm{h}} / \mathrm{y} / \mathrm{k}^{\mathrm{h}} /$ en $/ \mathrm{f} / \mathrm{y} / \chi /$, respectivamente [...]; los del centro (hablados en Tungurahua, Chimborazo, Cañar y Azuay) y sur (localizado en Loja) preservan la aspiración para las tres oclusivas. Por su parte la variedad sureña se distingue de las otras dos por mantener la /I//, pronunciada más bien como [1y] (Cerrón-Palomino, 1987, p. 240).

En el dialecto del centro habría que incluir Cotopaxi y excluir Cañar y Azuay, pues estos últimos son más afines con el de Saraguro (Loja). En cuanto a los dialectos de la Amazonía, el autor hace una comparación de 11 morfemas en los tres dialectos señalados por Carolyn Orr.

\section{Willen F. Adelaar con la colaboración de Peter Muysken}

Estos autores, en su libro The Languages of the Andes (2004), dieron importancia a los procesos morfofonémicos en la diversificación dialectal del quichua ecuatoriano, al manifestar que:

The Quechua dialects of Ecuador are morphologically, syntactically and lexically quite similar. The main differences lie in a number of morphophonological processes that affected the affixes in particular. While a southern dialect such as Cañar is rather conservative, Salasaca Quechua has undergone most of the processes involved (Adelaar y Muysken, 2004, p. 237).

Hay una coincidencia de Adelaar y Muysken (2004) con CerrónPalomino (1987) al considerar que las hablas de la Sierra sur son las más conservadoras, en tanto que el habla de Salasaca, que es parte de la Sierra centro, es la más innovadora. 
Figura 13

Realización del fonema $/ \lambda /$ en diferentes lugares

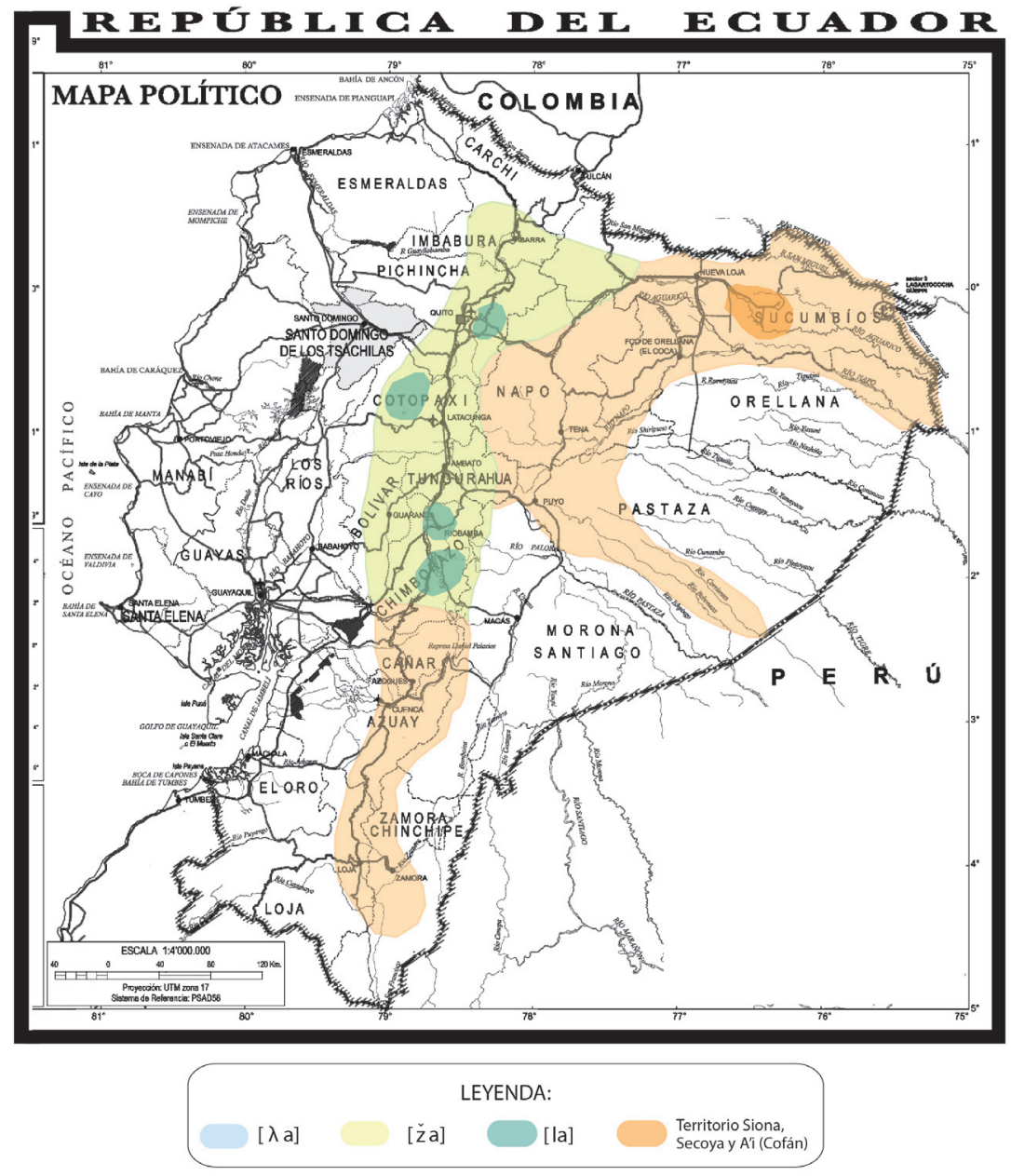

Fuente: el autor 